\title{
Is PARP-1 Inhibitor (Nutlin3a) Effective on Hepatic Ischemia-Reperfusion Injury?
}

\author{
Daisuke Kawahara ${ }^{1}$, Kosho Yamanouchi ${ }^{1}$, Naoyuki Okita ${ }^{2}$, Yoshikazu Higami ${ }^{2}$, Yusuke Inoue ${ }^{1}$, \\ Shinichiro Ito ${ }^{1}$, Masaaki Hidaka ${ }^{1}$, Kengo Kanetaka ${ }^{1}$, Fumihiko Fujita ${ }^{3}$, Susumu Eguchi ${ }^{{ }^{*}}$
}

'Department of Surgery, Nagasaki University Graduate School of Biomedical Sciences, 1-7-1 Sakamoto, Nagasaki, 852-8501, Japan

${ }^{2}$ Laboratory of Molecular Pathology and Metabolic Disease, Faculty of Pharmaceutical

Sciences, Tokyo University of Science, 2641 Yamazaki, Noda-shi, Chiba 278-0022, Japan

${ }^{3}$ Department of Surgery, Kurume University, 67 Asahi, Kurume, 830-0011, Japan

\section{ABSTRACT}

Background/Aims: Nutlin3a is known to have effects of stabilization of p53 and degradation of Poly(ADP-ribose) polymerase 1 (PARP1) in cell-specific manner. It was demonstrated that nutrlin3a decrease tissue damage caused by ischemia reperfusion (I/R) in transplant settings such as lung. We aimed to evaluate if the reagent would still alleviate hepatic I/R injury.

Methodology: First, to evaluate an efficacy of nutlin3a in normal liver, we administered it to male C57BI/6 mice intraperitoneally at doses of 0 (vehicle only), 5 , and $20 \mathrm{mg} / \mathrm{kg}$ of body-weight. The animals were killed at 4, 8, and 12 hours after administration and proteins of p53 and PARP1 in the liver were evaluated. Next, we assessed the effects of nutlin3a on the liver after I/R. Twenty-four hours after administration of nutlin3a, the mice suffered from hepatic ischemia, and then were sacrificed at 8 and 24 hours after reperfusion.

Results: No changes were observed in proteins of p53 and PARP1 in both the normal and I/R liver by administration of nutlin3a. Nutilin3a did not only show the effect of alleviation of hepatic I/R injury, but also seemed to have cytotoxicity to hepatocyte in dose-dependent manner.

Conclusion: A protective effect of nutilin3a was not evident in hepatic I/R model.

Key words: PARP1, liver, ischemia-reperfusion injury

\section{INTRODUCTION}

Hepatic ischemia-reperfusion (I/R) frequently occurs following liver resection or transplantation, and can result in hepatic injury and dysfunction (1,2). Hepatic I/R injury likely involves inflammation and reactive oxygen species (ROS), which directly damage hepatocytes (3). Poly(ADP-ribose) polymerase 1 (PARP1) is a major enzyme catalyzing poly (ADP-ribosyl)ation, which is involved in replication, DNA repair, and cell death $(4,5)$. DNA breaks lead to PARP1 activation and then poly(ADP-ribosyl)ation is catalyzed on substrate proteins in DNA damage regions, which is required for efficient recruitment of DNA repair factors to the
${ }^{*}$ Corresponding author: Susumu Eguchi, MD, Ph.D. Department of Surgery, Nagasaki University Graduate School of Biomedical Sciences, 1-7-1 Sakamoto, Nagasaki, 852-8501, Japan

Phone $\quad+81958197316$

Fax +81958197319

E-mail: sueguchi@nagasaki-u.ac.jp

\section{Abbreviations:}

PARP1: Poly(ADP-ribose) polymerase 1 $\mathrm{I} / \mathrm{R}$ : ischemia-reperfusion ROS: reactive oxygen species SD: standard deviation GPT: glutamic-pyruvic transaminase

Received: 29.06 .2020 Accepted: 26.08.2020

Copyright $\odot$ Celsius Publishing House www.sgo-iasgo.com 
loci (6). In contrast, over-activation of PARP1 consumes cellular NAD+ and ATP, resulting in breakdown of energy metabolism and cellular necrosis $(7,8)$. I/R induces PARP1 over-activation by production of reactive oxygen species (9). PARP1 should be an attractive target of not only cancer chemotherapy but also protection from I/R injury and PARP1 inhibitors have been evaluated in animal experiments and clinical trials (10).

A tumor-suppressor p53 has versatile physiological roles including forming a cell cycle checkpoint and functioning in DNA repair, apoptosis, and energy metabolism (11). Because p53 upregulates anti-oxidant and anti-inflammatory genes $(12,13)$, stabilization of p53 would lead to protecting cells from I/R-induced damages. The biological activity of p53 is regulated by murine double minute-2 (MDM2), an E3 ligase, which is known to inactivate p53 via ubiquitination and proteasomal degradation.

The small molecular compound nutlin3a is an MDM2 inhibitor and is potent to stabilize p53 (14). Nutlin3a was associated with tubular cell healing during acute kidney injury or decrease in inflammation and tissue damage in lung induced by $I / R$ in animal experiments (15). On the other hand, nutlin3a also induces the proteasomal degradation of PARP1 in mouse fibroblast cell lines (16). Thus, nutlin3a has potentially protective effects on I/R injury, via multiple mechanisms, i.e., p53 stabilization and PARP1 degradation. In this preliminary study, we evaluated efficacies of nutlin3a on expression or activation of these 2 kinds of proteins and hepatic warm I/R injury in mice.

\section{METHODOLOGY}

\section{Animals}

Male C57BI/6 mice (Japan SLC, Inc., Hamamatsu, Japan) weighing 22 - $25 \mathrm{~g}$ were used. The animals were housed in a temperature-controlled environment with a 12-hr light/dark cycle and had free access to standard rat chow and water. All experiments were performed according to the Guidelines for Animal Experimentation at Nagasaki University.

\section{Surgical procedure}

Hepatic warm I-R was induced as previously reported (17). Briefly, animals were anesthetized with isoflurane inhalation for induction and maintenance. A midline laparotomy was made and the inflow vessels, including the hepatic arteries and the portal veins supplying the left and median lobes of the liver, were occluded with small vascular clamps. After 45 minutes of ischemia, the vessels were reperfused and the abdomen was then closed.

\section{Experimental design}

First, we evaluated the effects of nutlin3a on the expression of p53 and PARP1 proteins in the normal liver. Nutlin3a was dissolved in 50\% DMSO/ $50 \%$ phosphate buffered saline and administered intraperitoneally at doses of 0 (vehicle only), 5, 20, and 100 $\mathrm{mg} / \mathrm{kg}$ of body-weight in 6 mice respectively. Two animals in each group were killed at 4,8 , and 12 hours after administration, liver samples were taken, and immediately immersed in liquid nitrogen. Second, we addressed to evaluate the effect of nutlin3a on hepatic I-R injury. After 24 hours of intraperitoneal administration of nutlin $3 a$ at the doses mentioned above in $\mathbf{4 0}$ mice, animals suffered hepatic I-R. Blood and liver samples were collected and the animals were killed at 8 and 24 hours after reperfusion. Blood samples were drawn from the inferior vena cava. The left lobes of the liver immediately immersed in liquid nitrogen. The serum and liver samples were stored at $-80 \stackrel{\circ}{\circ}$ until analysis. The median lobes of the liver were sliced at a thickness of $5 \mathrm{~mm}$ and fixed with buffered formalin for histological examination.

\section{Biochemical analysis}

Serum levels of glutamic-pyruvic transaminase (GPT) levels were determined by enzymatic methods at SRL, Inc. (Tokyo, Japan).

\section{Western blot studies}

We carried out Western blot to see the effects of nutlin3a on expression of PARP1, p53, and apoptosis related protein, and lipid peroxidation. Ten micrograms of liver lysates were loaded on an SDS/glycine/5\%-12\% polyacrylamide gradient gel. Following electrophoresis, proteins were transferred onto a cellulose nitrate membrane (PALL Corppration, NY). Membranes were blocked with $2.5 \%$ Skim milk (WAKO Pure Chemical Industries, Ltd., Osaka, Japan ) / 0.25\% BSA in TTBS (25mM Tris- $\mathrm{HCl}$ pH7.4, $140 \mathrm{mM} \mathrm{NaCl}, 2.5 \mathrm{mM} \mathrm{KCl}, 0.1 \%$ Tween 20) followed by incubation with a antimouse p53 antibody (Merck, NJ) or a antirabbit PARP1 antibody (Cell Signaling Technology, Inc., MA) or a antirabbit Caspase-7 antibody (Cell Signaling Technology) or a antimouse 4-HNE antibody (Japan Institute for the Control of Aging, NIKKEN SEIL Co., LTD, Shizuoka, Japan). After washing with TTBS, membranes were incubated with horseradish peroxidase-conjugated 
$F\left(a b^{\prime}\right) 2$ fragment of goat anit-mouse IgG, Light Chain Specific or horseradish peroxidase - conjugated $F\left(a b^{\prime}\right) 2$ fragment of goat anit-rabbit IgG or horseradish peroxidase - conjugated $F\left(a b^{\prime}\right) 2$ fragment of goat anit-mouse IgG (Jackson Immunological Research Laboratories, Inc., PA) and developed using Western blotting detection reagents (ImmunoStar LD, WAKO).

\section{Statistical Analysis}

Results are expressed as a mean \pm standard deviation (SD). The one-way ANOVA was used for continuous variables among three groups. A p-value of less than 0.05 was considered statistically significant.

\section{RESULTS}

Effects of nutlin3a in the normal liver

Either p53 activation or PARP1 degradation was not seen at any time point after nutlin3a administration (fig. 1).

Effects of nutlin3a on the hepatic ischemicreperfusion injury

The serum ALT was elevated significantly at 8 hours after reperfusion and the values were not different dependent on the doses of nutlin3a (fig. 2). The values of ALT at 24 hours tended to be getting higher

p53 positive control

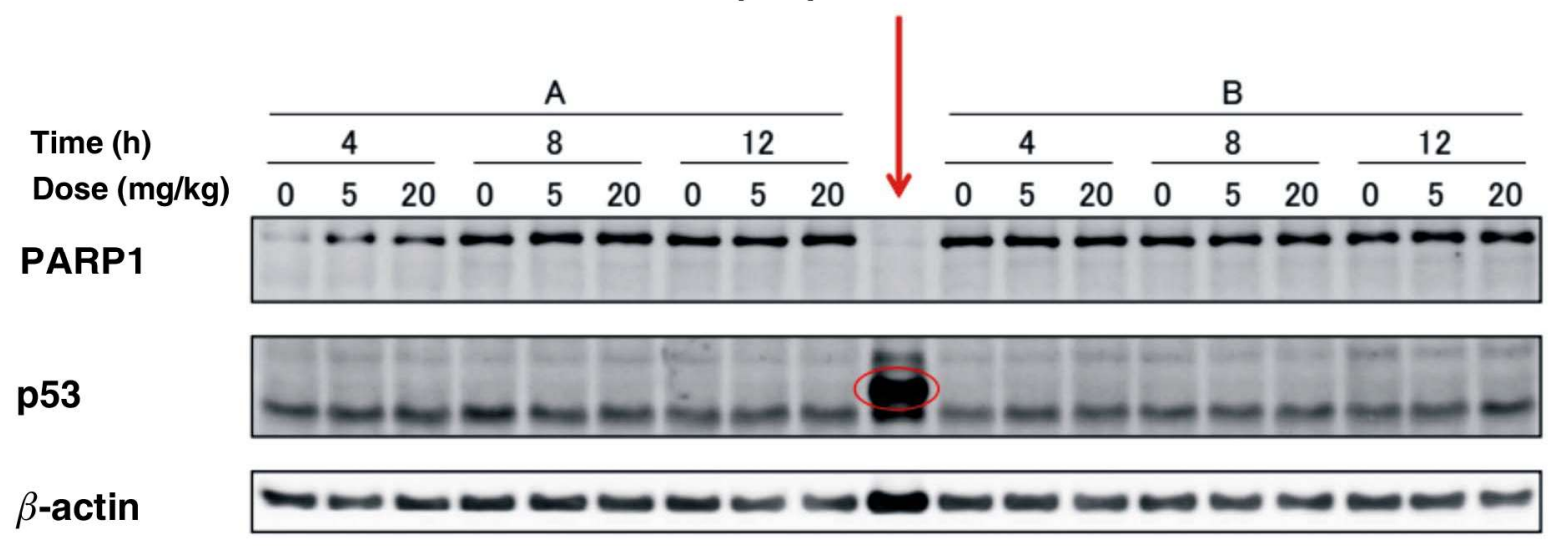

Figure 1 - Western blotting for p53 and PARP1 proteins in the normal liver after nutlin3a administration

(IU/I)

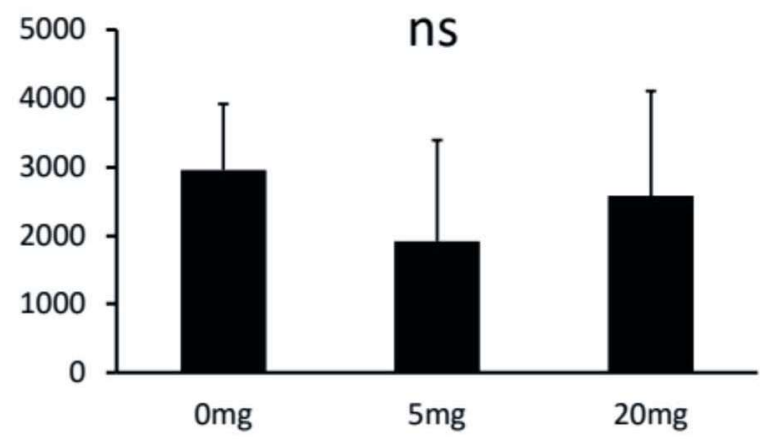

Dosage of nutlin3a

( $\mathrm{mg} / \mathrm{kg}$ body weight)

8 hours
(IU/I)

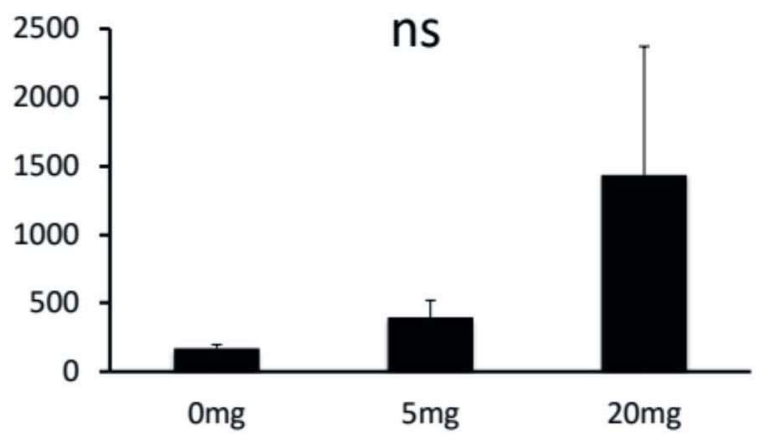

Dosage of nutlin3a

( $\mathrm{mg} / \mathrm{kg}$ body weight)

24 hours

Figure 2 - Serum levels of ALT at 8 and 24 hours after reperfusion ( $n=5$ each). Values are mean $+/-$ standard deviation ns; not significant 


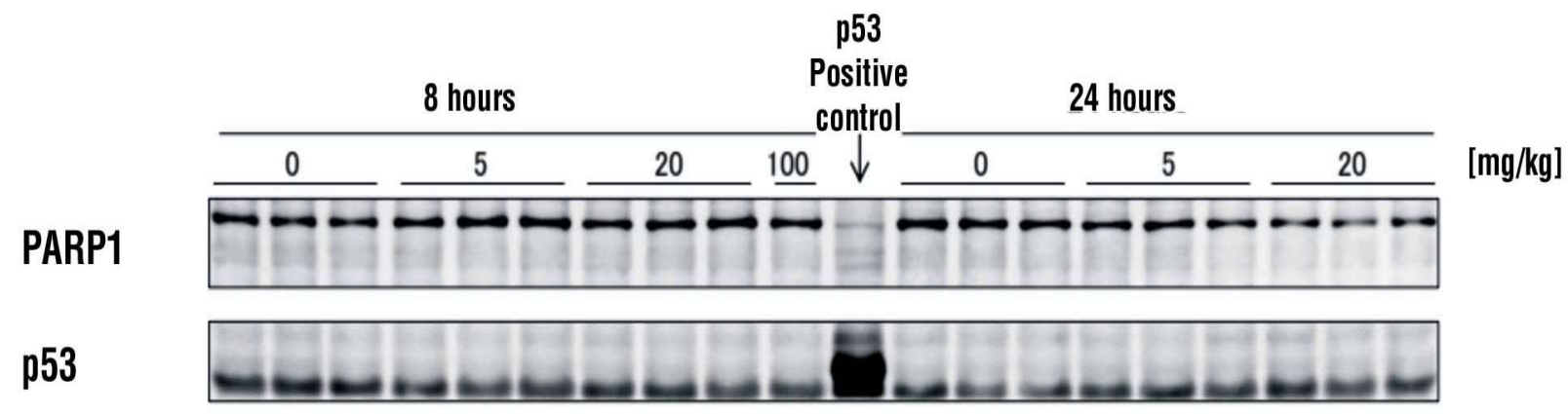

Figure 3. Western blotting for p53, PARP1, Caspase-7, and 4-HNE proteins in the liver after ischemia reperfusion. 4-HNE; 4-hydroxy-2-nonenal

dependent of doses of nutlin3a ( $p=0.07 .168,396,1436$ IU/I each for 0,5 , 20mg of nutlin3a).

In Western blotting, nutlin3a did not affect the expression of either p53 or PARP1 at 8 and 24 hours after reperfusion (fig. 3). Although cleaved forms of Caspase-7 were identified faintly at 8 hours after reperfusion, whose intensity was identical among the groups, they disappeared at 24 hours. Expression of 4-HNE was obvious at both 8 and 24 hours after reperfusion with nutlin3a administration compared with vehicle only.

\section{DISCUSSION}

Nutlin3a did not induce degradation of PARP1 and activate $\mathrm{p} 53$ in either normal or I/R liver in vivo. One possible reason of the results is cell-selective effect of nutlin3a, which was demonstrated to be in vitro experiments, i.e., positive in 3T3-F442A (mice fibroblast) and $A 549$ (human lung cancer) and negative in U2OS (human osteosarcoma) and HepG2 (human hepatoma) (16). We should carry out the experiment on effect of nutlin3a on hepatocytes in vitro to elucidate this hypothesis.

We administered nutlin3a intraperitoneally at 24 hours before hepatic $\mathrm{I} / \mathrm{R}$, following the protocol reported previously (17), where they demonstrated the effects of ntlin3a on acute kidney injury induced by I/R. Nutlin3a was, however, known to be catalyzed and almost disappeared within 24 hours of oral administration and PARP1 degraded by nutlin3a recovered quickly (18). So in our experiment, even if PARP1 was degraded in hepatocyte, it could recover to basal level by our observed time-points.

Cleaved Caspase-7, which was a activated form, was seen faintly after 8 hours of reperfusion, but not after
24 hours. Caspase-7-specific activities and liver cell apoptosis was reported to increase in ischemic hepatic lobes at 3 and 6 hours after hepatic I/R in rats $(19,20)$. These findings might indicate that activation of $p 53$ or degradation of PARP1 occurred before 8 hours of reperfusion, leading to only faint apoptotic signal observed after 8 hours in our experiment.

Nutlin3a itself seemed to enhance oxidative stress in the liver after $\mathrm{I} / \mathrm{R}$, as shown by expression of 4-HNE. Serum ALT tended to get higher with increasing doses of given nutlin3a after 24 hours of reperfusion. Moreover, although pathological damage seemed to alleviate after 8 hours of reperfusion by administration of nutlin3a at a dose of $5 \mathrm{mg} / \mathrm{kg}$, it was deteriorated with high dosage $(20 \mathrm{mg} / \mathrm{kg})$ of nutlin3a. These results implied that nutlin3a itself had toxicity to the liver in a dose-dependent manner and we have to inject this reagent to normal animals and evaluate liver injury to elucidate the premise.

\section{CONCLUSION}

We could not demonstrate the effect of nutlin3a on expressions of either p53 or PARP1 in the liver at time-points at which we investigated. Moreover, nutlin3a itself seemed to have cytotoxicity. To elucidate conclusive effects of nutlin3a in the liver, shorter interval between administration and evaluation, and less dosage of the reagent would be study points in the future.

\section{Ethical approval}

All procedures performed in studies involving human participants were in accordance with the ethical standards of the institutional and/or national research 
committee and with the 1964 Helsinki Declaration and its later amendments or comparable ethical standards.

\section{Conflict of interest}

The authors declare no conflict of interest in association with this study.

\section{REFERENCES}

1. Akhtar MZ, Sutherland Al, Huang H, Ploeg RJ, Pugh CW. The role of hypoxia-inducible factors in organ donation and transplantation: the current perspective and future opportunities. Am J Transplant 2014; 14(7):1481-1487.

2. Fu H, Xu H, Chen H, Li Y, Li W, Zhu Q, et al. Inhibition of glycogen synthase kinase 3 ameliorates liver ischemia/reperfusion injury via an energy-dependent mitochondrial mechanism. J Hepatol 2014; 61(4): 816-824.

3. Yamanouchi K, Eguchi S, Kamohara Y, Yanaga K, Okudaira S, Tajima $\mathrm{Y}$, et al. Glycine reduces hepatic warm ischaemia-reperfusion injury by suppressing inflammatory reactions in rats. Liver Int 2007; 27(9): 1249-1254.

4. Matsutani M, Nakagama H, Sugimura T. Poly(ADP-ribosyl)ation in relation to cancer and autoimmune disease. Cell Mol Life Sci 2005; 62(7-8): 769-783.

5. Miwa M, Matsutani M. PolyADP-ribosylation and cancer. Cancer Sc 2007; 98(10): 1528-1535.

6. Mortusewaicz 0 , Ame JC, Schreiber V, Leonhardt H. Feedbackregulated poly(ADP-ribosyl)ation by PARP-1 is required for rapid response to DNA damage in living cells. Nucleic Acids Res 2007; 35(22): 7665-7675

7. Ha HC, Snyder SH. Poly(ADP-ribose) polymerase is a mediator of necrotic cell death by ATP depletion. Proc Natl Acad Sci U S A 1999; 96(24): 13978-13982.

8. Herceg Z, Wang ZQ. Failure of poly(ADP-ribose) polymerase cleavage by caspases leads to induction of necrosis and enhanced apoptosis. Mol Cell Biol 1999:19(7): 5124-5133.

9. Pacher P, Szabo C. Role of the peroxynitrite-poly(ADP-ribose) polymerase pathway in human disease. Am J Pathol 2008; 173(1): 2-13.

10. Ferraria DV. Evolution of poly(ADP-ribose) polymerase-1 (PARP-1) inhibitors. From concept to clinic. J Med Chem 2010; 53(12): 45614584

11. Green DR, Kroemer G. Cytoplasmic functions of the tumour suppressor p53. Nature 2009; 458(7242): 1127-1130.

12. Sablina AA, Budanov AV, llyinskaya GV, Agapovaa LS, Kravchenko $\mathrm{JE}$, Chumakov PM. The antioxidant function of the p53 tumor suppressor. Nat Med 2005; 11(12): 1306-1313.

13. Komarova EA, Krivokrysenko V, Wang K, Neznanov N, Chernov MV, Komarov PG, et al. p53 is a suppressor of inflammatory response in mice. FASEB J 2005; 19(8): 1030-1032.

14. Mulay SR, Thomasova D, Ryu M, Anders HJ. MDM2 (murine double minute-2) links inflammation and tubular cell healing during acute kidney injury in mice. Kidney Int 2012; 81(12): 1199-1211.

15. Hatachi G, Tsuchiya T, Miyazaki T, Tsuchiya T, Taniguchi D, Doi R, et al. The Poly(Adenosine Diphosphate-Ribose) polymerase inhibitor PJ34 reduces pulmonary ischemia-reperfusion injury in rats. Transplantation 2014; 98(6): 618-623.

16. Matsushima S, Okita N, Oku M, Nagai W, Kobayashi M, Higami Y. An Mdm2 antagonist, Nutlin-3a, induces p53-dependent and proteasome-mediated poly(ADP-ribose) polymerase1 degradation in mouse fibroblasts. Biochem Biophys Res Commun 2011; 407(3): 557-561.

17. Lentsch AB, Yoshidome H, Cheadle WG, Miller FN, Edwardse MJ. Chemokine involvement in hepatic ischemia/reperfusion injury in mice: roles for macrophage inflammatory protein-2 and KC. Hepatology 1998; 27(2): 1172-1177.

18. Zhang F, Tagen M, Throm S, Mallari J, Miller L, Guy RK, et al. Wholebody physiologically based pharmacokinetic model for nutlin-3a in mice after intravenous and oral administration. Drug Metab Dispos 2011; 39(1): 15-21

19. Cursio R, Colosetti P, Auberger P, Gugenheim J. Liver apoptosis following normothermic ischemia-reperfusion: in vivo evaluation of caspase activity by FLIVO assay in rats. Transplant Proc 2008; 40(6): 2038-2041.

20. Cursio R, Colosetti P, Saint-Paul MC, Pagnotta S, Gounon P, lannelli $A$, et al. Induction of different types of cell death after normothermic liver ischemia-reperfusion. Transplant Proc 2010; 42(10): 39773980 